\title{
A Special class of Hypersurfaces parametrized by lines of curvature in $\mathbb{R}^{4}$
}

\section{Una clase especial de Hipersuperficies parametrizadas por líneas de curvatura en $\mathbb{R}^{4}$}

\author{
Carlos M.C. Riveros *
}

Received, Feb. 08, 2018

Accepted, May. 31, 2018

DOI: http://dx.doi.org/10.17268/sel.mat.2018.01.07

\begin{abstract}
In this paper we study hypersurfaces in $\mathbb{R}^{4}$ parametrized by lines of curvature with three distinct principal curvatures and with Laplace invariants $m_{j i}=m_{k i}=0, m_{j i k} \neq 0$ for $i, j, k$ distinct fixed indices. We characterize locally a generic family of such hypersurfaces in terms of the principal curvatures and three vector valued functions of one variable, this family includes a classe of Dupin hypersurfaces. Moreover, we show that these vector valued functions are invariant under inversions and homotheties.
\end{abstract}

Keywords Dupin hypersurfaces, Laplace invariants, lines of curvature

\section{Resumen}

En este artículo estudiamos hipersuperficies en $\mathbb{R}^{4}$ parametrizadas por líneas de curvatura con tres curvaturas principales distintas y con invariantes de Laplace $m_{j i}=m_{k i}=0, m_{j i k} \neq 0$ para indices fijos $i, j, k$ distintos. Caracterizamos localmente una familia genérica de tales hipersuperficies en términos de las curvaturas principales y tres funciones vectoriales de una variable, esta familia incluye una clase de hipersuperficies de Dupin. Ademas, mostramos que estas funciones vectoriales son invariantes por inversiones y dilataciones.

Palabras clave. Hipersuperficies de Dupin, Invariantes de Laplace, Líneas de curvatura

1. Introduction. Dupin surfaces were first studied by Dupin in 1822 and more recently by many authors [1]-[6], [9]-[14] and [16],[17], which studied several aspects of Dupin hypersurfaces. The class of Dupin hypersurfaces is invariant under Lie transformations [11]. Therefore, the classification of Dupin hypersurfaces is considered up to these transformations. The local classification of Dupin surfaces in $\mathbb{R}^{3}$ is well known. Pinkall [12] gave a complete classification up to Lie equivalence for Dupin hypersurfaces $M^{3} \subset \mathbb{R}^{4}$, with three distinct principal curvatures. Niebergall [10] and more recently Cecil and Jensen [6] studied proper Dupin hypersurfaces with four distinct principal curvatures and constant Lie curvature (the cross-ratio of four principal curvatures).

Riveros [15] obtained a local characterization of the Dupin hypersurfaces in $\mathbb{R}^{4}$ parametrized by lines of curvature, with three distinct principal curvatures and $m_{j i k} \neq 0$, in terms of the principal curvatures and three vector valued functions in $\mathbb{R}^{4}$ which are invariant under inversions and homotheties, in this case the Laplace invariants $m_{i j}=0$, for $1 \leq i \neq j \leq 3$.

In this paper we study generic hypersurfaces in $\mathbb{R}^{4}$, parametrized by lines of curvature, with three distinct principal curvatures and with Laplace invariants $m_{j i}=m_{k i}=0, m_{j i k} \neq 0$. We obtain a local characterization of a generic family of such hypersurfaces (Theorem 3.1), in terms of the principal curvature functions and three vector valued functions of one variable. This family of hypersurfaces includes the Dupin

*Departamento de Matemática, Universidade de Brasília, 70910-900, Brasília-DF, Brazil. carlos@mat.unb.br. 
hypersurfaces studied by Riveros [15]. The characterization is based on the theory of higher-dimensional Laplace invariants introduced by Kamran-Tenenblat [7]-[8].

In section 2, we give some properties of hypersurfaces with distinct principal curvatures. In section 3 , Theorem 3.1 gives a local characterization of generic hypersurfaces in $\mathbb{R}^{4}$ with three distinct principal curvatures. Moreover, we show that the vector valued functions, which appear in the characterization of Theorem 3.1 are invariant under inversions and homotheties, but the functions are not invariant under isometries. Therefore, the vector valued functions are not invariant under the full group of Lie transformations of $\mathbb{R}^{4}$.

2. Preliminaries. Let $\Omega$ be an open subset of $\mathbb{R}^{n}$ and $x=\left(x_{1}, x_{2}, \cdots, x_{n}\right) \in \Omega$. Let $X: \Omega \subset \mathbb{R}^{n} \rightarrow$ $\mathbb{R}^{n+1}, \quad n \geq 3$, be a hypersurface parametrized by lines of curvature, with distinct principal curvatures $\lambda_{i}, 1 \leq i \leq n$ and $N: \Omega \subset \mathbb{R}^{n} \rightarrow \mathbb{R}^{n+1}$ be a unit normal vector field of $X$. Then

$$
\begin{aligned}
\left\langle X_{, i}, X_{, j}\right\rangle & =\delta_{i j} g_{i i}, 1 \leq i, j \leq n, \\
N_{, i} & =-\lambda_{i} X_{, i},
\end{aligned}
$$

where the subscript ${ }_{, i}$ denotes the derivative with respect to $x_{i}$. Moreover,

$$
\begin{aligned}
& X_{, i j}-\Gamma_{i j}^{i} X_{, i}-\Gamma_{i j}^{j} X_{, j}=0, \quad 1 \leq i \neq j \leq n, \\
& \Gamma_{i j}^{i}=\frac{\lambda_{i, j}}{\lambda_{j}-\lambda_{i}}, \quad 1 \leq i \neq j \leq n,
\end{aligned}
$$

where $\Gamma_{i j}^{k}$ are the Christoffel symbols.

We now consider the higher-dimensional Laplace invariants of the system of equations (2.2) (see [7]-[8] for the definition of these invariants),

$$
\begin{aligned}
m_{i j} & =-\Gamma_{i j, i}^{i}+\Gamma_{i j}^{i} \Gamma_{i j}^{j}, \\
m_{i j k} & =\Gamma_{i j}^{i}-\Gamma_{k j}^{k}, \quad k \neq i, j, \quad 1 \leq k \leq n .
\end{aligned}
$$

As a consequence of (2.3) and the un-numbered lemma appearing in [8], we obtain the following identities, valid for distinct $i, j, k, l, 1 \leq i, j, k, l \leq n$ :

$$
\begin{aligned}
m_{i j k}+m_{k j i} & =0, \\
m_{i j k, k}-m_{i j k} m_{j k i}-m_{k j} & =0, \\
m_{i j, k}+m_{i j k} m_{i k}+m_{i k j} m_{i j} & =0 \\
m_{i j k}-m_{i j l}-m_{l j k} & =0 \\
m_{l i k, j}+m_{i j l} m_{k i l}+m_{l j k} m_{k i j} & =0 .
\end{aligned}
$$

From Remark 2.2 in [16], follows that for $n \geq 3$, the higher-dimensional Laplace invariants do not change under inversions in spheres centered at the origin and homotheties.

For hypersurfaces with distinct principal curvatures, the Möbius curvature is defined, for distinct $i, j, k$, by

$$
C^{i j k}=\frac{\lambda_{i}-\lambda_{j}}{\lambda_{k}-\lambda_{j}}
$$

Since all $\lambda_{i}$ are distinct we conclude that $C^{i j k} \neq 0$ and $C^{i j k} \neq 1$. Möbius curvatures are invariant under Möbius transformations.

The following result was obtained in [14], which provides some properties which are satisfied by the principal curvatures of a hypersurface in $\mathbb{R}^{n+1}$ parametrized by lines of curvature.

LEMMA 1. Let $\lambda_{r}: \Omega \subset \mathbb{R}^{n} \rightarrow \mathbb{R}, n \geq 3$, be smooth functions distinct at each point. Consider functions $m_{i j k}$ defined by (2.3) and (2.4). Then for $i, j$ fixed, $1 \leq i \neq j \leq n$, the following properties hold

$$
\begin{aligned}
& {\left[C^{k j i} m_{j k i}\right]_{, i}=m_{j k i, i}+\left[\frac{\lambda_{i, i}}{\lambda_{j}-\lambda_{i}}\right]_{, k},} \\
& {\left[C^{k j i} m_{j k i}\right]_{, j}=-\left[\frac{\lambda_{j, j}}{\lambda_{j}-\lambda_{i}}\right]_{, k},} \\
& {\left[C^{k j i} m_{j k i}\right]_{, l}=\left[C^{l j i} m_{j l i}\right]_{, k},}
\end{aligned}
$$


where $C^{k j i}$ is the Möbius curvature and $1 \leq k \neq l \leq n$ are distinct from $i$ and $j$. The following Lemma is a application of Theorem 1 in [8].

LEMMA 2. Let $X: \Omega \subset \mathbb{R}^{n} \rightarrow \mathbb{R}^{n+1}, n \geq 3$, be a hypersurface parametrized by lines of curvature, with $n$ distinct principal curvatures $\lambda_{r}, \quad 1 \leq r \leq n$. For $i, j, k$ fixed, $1 \leq i \neq j \neq k \leq n$, the transformation

$$
X=V \bar{X}, \text { where } \quad V=\frac{e^{-\int C^{k j i} m_{j k i} d x_{k}}}{\lambda_{j}-\lambda_{i}},
$$

transforms system (2.2) into

$$
\begin{aligned}
\bar{X}_{, i j}+A \bar{X}_{, j}-m_{i j} \bar{X} & =0, \\
\bar{X}_{, i r}+\left(A+m_{j i r}\right) \bar{X}_{, r}-m_{i r} \bar{X} & =0, \\
\bar{X}_{, j r}+m_{i r j} \bar{X}_{, j}+m_{i j r} \bar{X}_{, r} & =0, \\
\bar{X}_{, r l}+m_{i l r} \bar{X}_{, r}+m_{i r l} \bar{X}_{, l} & =0,
\end{aligned}
$$

where $l$ and $r$ are such that $1 \leq r \neq l \neq i \neq j \leq n$ and

$$
A=-\int m_{j k i, i} d x_{k}
$$

Moreover,

$$
A_{, j}=m_{j i}-m_{i j}, A_{, r}=-m_{j r i, i} .
$$

REMARK 1. For subsequent use, we will compute the derivatives of the function $V$ given by (2.10). It follows from Lemma 1 that,

$$
\begin{aligned}
V_{, i} & =\left(A+\Gamma_{j i}^{j}\right) V, \\
V_{, j} & =\Gamma_{i j}^{i} V, \\
V_{, k} & =\Gamma_{i k}^{i} V, \\
V_{, l} & =\Gamma_{i l}^{i} V,
\end{aligned}
$$

where $A$ is given by (2.12) and $l$ is distinct from $i, j, k$.

3. Main results. In this section, we prove our main result which provides a local characterization of generic hypersurfaces parametrized by lines of curvature in $\mathbb{R}^{4}$, with three distinct principal curvatures. We remark that in the case of Dupin hypersurfaces parametrized by lines of curvature the Laplace invariants $m_{i j}=0$, for $1 \leq i \neq j \leq 3$ and therefore, this family of hypersurfaces includes the class of Dupin hypersurfaces studied by Riveros in [15].

THEOREM 1. Let $X: \Omega \subset \mathbb{R}^{3} \rightarrow \mathbb{R}^{4}$, be a hypersurface parametrized by lines of curvature, with three distinct principal curvatures $\lambda_{r}$. For $i, j, k$ distinct fixed indices, suppose $m_{j i}=m_{k i}=0$ and $m_{j i k} \neq 0$ then

$$
X=V\left[B_{j}-B_{k}\right],
$$

where

$$
V=\frac{e^{-\int C^{k j i} m_{j k i} d x_{k}}}{\lambda_{j}-\lambda_{i}}, \quad B_{s}=\frac{1}{Q_{s}}\left[\int \frac{Q_{s} G_{i}\left(x_{i}\right)}{m_{j i k}} d x_{i}+G_{s}\left(x_{s}\right)\right], s \neq i,
$$

$G_{r}\left(x_{r}\right), r=i, j, k$, are vector valued functions of $\mathbb{R}^{4}, A_{, j}=-m_{i j}, A=-\int m_{j k i, i} d x_{k}$ and

$$
Q_{s}=\left\{\begin{array}{l}
e^{\int A d x_{i}} \text { if } s=j, \\
e^{\int\left(A+m_{j i s}\right) d x_{i}} \text { if } s=k .
\end{array}\right.
$$


Moreover, considering

$$
\alpha^{i}=\left(A+\frac{\lambda_{j, i}}{\lambda_{i}-\lambda_{j}}\right) M+M_{, i}, \quad \alpha^{s}=\frac{\lambda_{i, s}}{\lambda_{s}-\lambda_{i}} M+M_{, s}, \quad s \neq i,
$$

where $M=B_{j}-B_{k}$, the functions $G_{r}\left(x_{r}\right)$ satisfy the following properties in $\Omega$, for $1 \leq r \neq t \leq 3$ :

a) $\alpha^{r} \neq 0$,

b) $\left\langle\alpha^{r}, \alpha^{t}\right\rangle=0, \quad r \neq t$

c) $\lambda_{r}=\frac{\left\langle\alpha_{, r}^{r}, \alpha^{i} \times \alpha^{j} \times \alpha^{k}\right\rangle}{V\left|\alpha^{r}\right|^{2}\left|\alpha^{i}\right|\left|\alpha^{j}\right|\left|\alpha^{k}\right|}$.

Conversely, let $\lambda_{r}: \Omega \subset \mathbb{R}^{3} \rightarrow \mathbb{R}, r=1,2,3$ be real functions, distinct at each point. Assume that the functions $m_{r t s}$ and $m_{r t}$ defined by

$$
\begin{aligned}
m_{r t s} & =\frac{\lambda_{r, t}}{\lambda_{t}-\lambda_{r}}-\frac{\lambda_{s, t}}{\lambda_{t}-\lambda_{s}}, 1 \leq r \neq t \neq s \leq 3, \\
m_{r t} & =-\left[\frac{\lambda_{r, t}}{\lambda_{t}-\lambda_{r}}\right]_{, r}-\frac{\lambda_{r, t} \lambda_{t, r}}{\left(\lambda_{t}-\lambda_{r}\right)^{2}}, 1 \leq r \neq t \leq 3,
\end{aligned}
$$

satisfy (2.5), and for $i, j, k$ distinct fixed indices, $m_{j i}=m_{k i}=0$, and $m_{j i k} \neq 0$. Then for any vector valued functions $G_{r}\left(x_{r}\right)$ satisfying properties a) b) c), where $\alpha^{r}$ is defined by (3.4), the function $X: \Omega \subset$ $\mathbb{R}^{3} \rightarrow \mathbb{R}^{4}$ given by (3.1) describes a hypersurface parametrized by lines of curvature whose principal curvatures are the functions $\lambda_{r}$.

Proof: We observe that from (2.5), the conditions $m_{j i}=m_{k i}=0$, implies that $m_{j k}=m_{k j}=0$. From equation (2.2) we have,

$$
X_{, s r}-\Gamma_{s r}^{s} X_{, s}-\Gamma_{s r}^{r} X_{, r}=0,1 \leq s \neq r \leq 3 .
$$

For fixed distinct indices $i, j, k$, we consider the transformation

$$
X=V \bar{X}
$$

as in Lemma 2, where $V$ is given by (2.10). Then system (3.6) reduces to

$$
\begin{gathered}
\bar{X}_{, i j}+A \bar{X}_{, j}-m_{i j} \bar{X}=0 \\
\bar{X}_{, i k}+\left(A+m_{j i k}\right) \bar{X}_{, k}-m_{i k} \bar{X}=0 \\
\bar{X}_{, j k}+m_{i k j} \bar{X}_{, j}+m_{i j k} \bar{X}_{, k}=0
\end{gathered}
$$

where

$$
A_{, j}=-m_{i j}, \quad A_{, k}=-m_{j k i, i} .
$$

It follows from the third and second equations of (2.5) and (3.9) that

$$
\left(A+m_{j i k}\right)_{, k}=-m_{i k}
$$

Using (3.9) and (3.10) in the first two equations of (3.8), we have that

$$
\begin{aligned}
\bar{X}_{, i}+A \bar{X} & =W^{j}\left(x_{i}, x_{k}\right), \\
\bar{X}_{, i}+\left(A+m_{j i k}\right) \bar{X} & =W^{k}\left(x_{i}, x_{j}\right),
\end{aligned}
$$

where $W^{j}$ and $W^{k}$ are functions that do not depend on $x_{j}$ and $x_{k}$, respectively. Since $m_{j i k} \neq 0$, from (3.11) and (3.12) we have

$$
\bar{X}=\frac{1}{m_{j i k}}\left[W^{k}-W^{j}\right] .
$$


Differentiating (3.13) and using (2.5) we have

$$
\begin{aligned}
& \bar{X}_{, i}=-\frac{m_{j i k, i}}{\left(m_{j i k}\right)^{2}}\left[W^{k}-W^{j}\right]+\frac{1}{m_{j i k}}\left[W^{k}-W^{j}\right]_{, i} \\
& \bar{X}_{, j}=\frac{m_{k j i}}{m_{j i k}}\left[W^{k}-W^{j}\right]+\frac{1}{m_{j i k}} W_{, j}^{k} \\
& \bar{X}_{, k}=\frac{m_{j k i}}{m_{j i k}}\left[W^{k}-W^{j}\right]-\frac{1}{m_{j i k}} W_{, k}^{j} \\
& \bar{X}_{, i j}=\frac{1}{m_{j i k}} W_{, i j}^{k}-\frac{m_{j i k, i}}{\left(m_{j i k}\right)^{2}} W_{, j}^{k}+\left[m_{k j i}+\frac{m_{i j}}{m_{j i k}}+\frac{m_{i j k} m_{j i k, i}}{\left(m_{j i k}\right)^{2}}\right]\left[W^{k}-W^{j}\right]+ \\
& -\frac{m_{i j k}}{m_{j i k}}\left[W^{k}-W^{j}\right]_{, i} \\
& \bar{X}_{, i k}=-\frac{1}{m_{j i k}} W_{, i k}^{j}+\frac{m_{j i k, i}}{\left(m_{j i k}\right)^{2}} W_{, k}^{j}+\left[m_{i k j}+\frac{m_{i k}}{m_{j i k}}+\frac{m_{i k j} m_{j i k, i}}{\left(m_{j i k}\right)^{2}}\right]\left[W^{k}-W^{j}\right]+ \\
& \text { (3.18) } \quad-\frac{m_{i k j}}{m_{j i k}}\left[W^{k}-W^{j}\right]_{, i} \\
& \text { (3.19) } \quad \bar{X}_{, j k}=\frac{2 m_{i j k} m_{i k j}}{m_{j i k}}\left[W^{k}-W^{j}\right]+\frac{m_{i j k}}{m_{j i k}} W_{, k}^{j}-\frac{m_{i k j}}{m_{j i k}} W_{, j}^{k} \text {. }
\end{aligned}
$$

The substitution of $\bar{X}$ and $\bar{X}_{, i}$ into (3.11) and (3.12), gives

$$
\begin{array}{r}
\left(A-\frac{m_{j i k, i}}{m_{j i k}}\right)\left[W^{k}-W^{j}\right]+\left[W^{k}-W^{j}\right]_{, i}=m_{j i k} W^{j}, \\
\left(A+m_{j i k}-\frac{m_{j i k, i}}{m_{j i k}}\right)\left[W^{k}-W^{j}\right]+\left[W^{k}-W^{j}\right]_{, i}=m_{j i k} W^{k} .
\end{array}
$$

Using (3.13), (3.15), (3.17) and (3.21 in first equation of system (3.8), we obtain

$$
W_{, i j}^{k}+\left(A-\frac{m_{j i k, i}}{m_{j i k}}\right) W_{, j}^{k}+m_{k j i} m_{j i k}=0,
$$

Using (3.13), (3.16), (3.18) and (3.20 in second equation of system (3.8), we obtain

$$
W_{, i k}^{i}+\left(A+m_{j i k}-\frac{m_{j i k, i}}{m_{j i k}}\right) W_{, k}^{j}+m_{i k j} m_{j i k}=0,
$$

Using (3.15), (3.16) and (3.19) in the third equation of system (3.8) we obtain an identity. From (2.5) and (3.9) we obtain

$$
\left(A-\frac{m_{j i k, i}}{m_{j i k}}\right)_{, j}=m_{k j i} m_{j i k},\left(A+m_{j i k}-\frac{m_{j i k, i}}{m_{j i k}}\right)_{, k}=m_{i k j} m_{j i k} .
$$

It follows from (3.2), (3.3) and (3.24) that the solutions of equations (3.22) and (3.23) are given by

$$
\begin{aligned}
W^{k}\left(x_{i}, x_{j}\right) & =\frac{m_{j i k}}{Q_{j}}\left[\int \frac{Q_{j} G_{i}\left(x_{i}\right)}{m_{j i k}} d x_{i}+G_{j}\left(x_{j}\right)\right] \\
W^{j}\left(x_{i}, x_{k}\right) & =\frac{m_{j i k}}{Q_{k}}\left[\int \frac{Q_{k} \tilde{G}_{i}\left(x_{i}\right)}{m_{j i k}} d x_{i}+G_{k}\left(x_{k}\right)\right]
\end{aligned}
$$

From (3.20), (3.25) and (3.26) it follows that

$$
G_{i}\left(x_{i}\right)=\tilde{G}_{i}\left(x_{i}\right)
$$

The substitution of (3.25), (3.26) and (3.27) in (3.13) gives

$$
\bar{X}=B_{j}-B_{k}
$$

where we have used (3.2), which substituted into (3.7), implies (3.1). 
Considering $\alpha^{i}$ and $\alpha^{s}, s=j, k$ defined by (3.4), it follows from (2.7), (2.8), (2.13) and (2.4) that

$$
X_{, r}=V \alpha^{r}, r=i, j, k .
$$

Differentiating (3.28), we have

$$
X_{, r r}=V_{, r} \alpha^{r}+V \alpha_{, r}^{r}, r=i, j, k .
$$

It follows from (3.28) that the metric of $X_{, r}$ is given by

$$
g_{r r}=(V)^{2}\left|\alpha^{r}\right|^{2}, g_{r t}=0, r \neq t .
$$

A unit vector field normal to $X$ is given by

$$
N=\frac{\alpha^{i} \times \alpha^{j} \times \alpha^{k} \times \alpha^{l}}{\left|\alpha^{i}\right|\left|\alpha^{j}\right|\left|\alpha^{k}\right|\left|\alpha^{l}\right|} .
$$

Since $X$ is a hypersurface parametrized by orthogonal curvature lines, with $\lambda_{s}$, as principal curvature we have, for $1 \leq r \neq s \leq 3$

$$
\left\langle N, X_{, r s}\right\rangle=0, \quad \lambda_{s}=\frac{\left\langle X_{, r r}, N\right\rangle}{g_{r r}}
$$

Hence from (3.29) and (3.31) we obtain for $r=i, j, k$,

$$
\lambda_{r}=\frac{\left\langle\alpha_{, r}^{r}, \alpha^{i} \times \alpha^{j} \times \alpha^{k} \times \alpha^{l}\right\rangle}{V\left|\alpha^{r}\right|^{2}\left|\alpha^{i}\right|\left|\alpha^{j}\right|\left|\alpha^{k}\right|\left|\alpha^{l}\right|} .
$$

Therefore, we conclude that conditions a), b) and c) are satisfied.

Conversely, let $\lambda_{r}$ be real functions distinct at each point. Assume that the functions $m_{r t s}$ and $m_{r t}$, defined by (3.5), satisfy (2.5) and suppose $G_{r}\left(x_{r}\right), 1 \leq r \leq 3$, are vector valued functions satisfying properties a), b) and c). Defining $X$ by (3.1), it follows from Lemma 1 and properties a) and b), that $X$ is an immersion, whose coordinates curves are orthogonal. Moreover, the induced metric is given by (3.30) and a unit normal vector field by (3.31).

Differentiating (3.28) with respect to $x_{t}$, using Lemma 1, the expressions (2.5), (2.14) and (3.4) we obtain

$$
X_{, r t}=V\left(\frac{\lambda_{r, t}}{\lambda_{t}-\lambda_{r}} \alpha^{r}+\frac{\lambda_{t, r}}{\lambda_{r}-\lambda_{t}} \alpha^{t}\right), r \neq t .
$$

From (3.31), it follows that $\left\langle X_{, r t}, N\right\rangle=0$. Hence the second fundamental form is diagonal and therefore the coordinates curves are lines of curvature. Moreover, it follows from (3.29) - (3.31) and from property c) that for $r=i, j, k$,

$$
\frac{\left\langle X_{, r r}, N\right\rangle}{g_{r r}}=\frac{\left\langle\alpha_{, r}^{r}, \alpha^{i} \times \alpha^{j} \times \alpha^{k} \times \alpha^{l}\right\rangle}{V\left|\alpha^{r}\right|^{2}\left|\alpha^{i}\right|\left|\alpha^{j}\right|\left|\alpha^{k}\right|\left|\alpha^{l}\right|}=\lambda_{r}
$$

which concludes the proof.

Now we show that the vector valued functions which appear in Theorem 1 are invariant under inversions and homotheties.

THEOREM 2. Let $X: \Omega \subset \mathbb{R}^{3} \rightarrow \mathbb{R}^{4}$ be a hypersurface with three distinct principal curvatures $\lambda_{r}$, parametrized by lines of curvature as in the Theorem 1. Then the vector valued functions $G_{r}\left(x_{r}\right), 1 \leq r \leq$ 3 are invariants under inversions and homotheties.

Proof: a) Assuming without loss of generality that $0 \notin X(\Omega)$, we consider $\tilde{X}=I^{4}(X)$ a hypersurface parametrized by lines of curvature, obtained by composing $X$ with the inversion defined by $I^{4}(X)=$ $\frac{X}{\langle X, X\rangle}$ and whose distinct principal curvatures are given by

$$
\tilde{\lambda}_{r}=\langle X, X\rangle \lambda_{r}+2\langle X, N\rangle, r=i, j, k .
$$

Applying the Theorem 1 to $\tilde{X}$, we have for $i, j, k$ fixed distinct indices

$$
\tilde{X}=\tilde{V}\left[\tilde{B}_{j}-\tilde{B}_{k}\right]
$$


where

$$
\begin{aligned}
\tilde{B}_{s} & =\frac{1}{\tilde{Q}_{s}}\left[\int \frac{\tilde{Q}_{s} \tilde{G}_{i}\left(x_{i}\right)}{\tilde{m}_{j i k}} d x_{i}+\tilde{G}_{s}\left(x_{s}\right)\right], s \neq i, \\
\tilde{V} & =\frac{e^{-\int \tilde{C}^{k j i} \tilde{m}_{j k i} d x_{k}}}{\tilde{\lambda}_{j}-\tilde{\lambda}_{i}}, \tilde{C}^{k j i}=\frac{\tilde{\lambda}_{k}-\tilde{\lambda}_{j}}{\tilde{\lambda}_{i}-\tilde{\lambda}_{j}} \\
\tilde{A} & =-\int \tilde{m}_{j k i, i} d x_{k}
\end{aligned}
$$

$\tilde{Q}_{s}, s \neq i \underset{\sim}{\operatorname{are}}$ defined by (3.3) and $\tilde{G}_{r}\left(x_{r}\right), r=i, j, k$ are vector valued functions in $\mathbb{R}^{4}$.

Since $\tilde{X}$ and $X$ have the same higher-dimensional Laplace invariants, we obtain

$$
\tilde{A}=A, \tilde{Q}_{s}=Q_{s}, s \neq i .
$$

Substituting (3.32) in (3.33), we have

$$
\tilde{V}=\frac{V}{\langle X, X\rangle}
$$

On the other hand,

$$
\tilde{X}=\frac{X}{\langle X, X\rangle}
$$

We will show that $\tilde{G}_{r}\left(x_{r}\right)=G_{r}\left(x_{r}\right), r=i, j, k$. It follows from (3.35) and (3.36) that

$$
\tilde{B}_{j}-B_{j}-\left(\tilde{B}_{k}-B_{k}\right)=0 .
$$

We observe that

$$
B_{j, i}=-A B_{j}+\frac{G_{i}\left(x_{i}\right)}{m_{j i k}}, \quad B_{k, i}=-\left(A+m_{j i k}\right) B_{k}+\frac{G_{i}\left(x_{i}\right)}{m_{j i k}} .
$$

This fact follows from the equalities

$$
Q_{j, i}=A Q_{j} \quad, \quad Q_{k, i}=\left(A+m_{j i k}\right) Q_{k} .
$$

Therefore differentiating (3.37) with respect to $x_{i}$, we get

$$
-A\left(\tilde{B}_{j}-B_{j}\right)+\left(A+m_{j i k}\right)\left(\tilde{B}_{k}-B_{k}\right)=0 .
$$

From (3.37) and the fact that $m_{j i k} \neq 0$, we have

$$
\tilde{B}_{k}=B_{k} .
$$

Differentiating with respect to $x_{i}$, we get $\tilde{G}_{k}\left(x_{k}\right)=G_{k}\left(x_{k}\right)$, hence it follows that $\tilde{G}_{i}\left(x_{i}\right)=G_{i}\left(x_{i}\right)$. Substituting (3.38) in (3.37), we obtain

$$
\tilde{B}_{j}=B_{j} .
$$

and hence $\tilde{G}_{j}\left(x_{j}\right)=G_{j}\left(x_{j}\right)$, which concludes the proof of a).

b) Let $\bar{X}=a X$ be a homothety of $X$. Then $\bar{X}$ is a hypersurface parametrized by lines of curvature, with distinct principal curvatures given by

$$
\bar{\lambda}_{r}=\frac{\lambda_{r}}{a}, \quad r=i, j, k
$$

Applying Theorem 1 to $\bar{X}$, we have for $i, j, k$ distinct fixed indices

$$
\bar{X}=\bar{V}\left[\bar{B}_{j}-\bar{B}_{k}\right]
$$


where

$$
\begin{aligned}
\bar{B}_{s} & =\frac{1}{\bar{Q}_{s}}\left[\int \frac{\bar{Q}_{s} \bar{G}_{i}\left(x_{i}\right)}{\bar{m}_{j i k}} d x_{i}+\bar{G}_{s}\left(x_{s}\right)\right], s \neq i, \\
\bar{V} & =\frac{e^{-\int \bar{C}^{k j i} \bar{m}_{j k i} d x_{k}}}{\bar{\lambda}_{j}-\bar{\lambda}_{i}}, \text { where } \bar{C}^{k j i}=\frac{\bar{\lambda}_{k}-\bar{\lambda}_{j}}{\bar{\lambda}_{i}-\bar{\lambda}_{j}} \\
\bar{A} & =-\int \bar{m}_{j k i, i} d x_{k},
\end{aligned}
$$

$\bar{Q}_{s}, s \neq i$ are defined by (3.3) and $\bar{G}_{r}\left(x_{r}\right), r=i, j, k$ are vector valued functions in $\mathbb{R}^{4}$.

Since $\bar{X}$ and $X$ have the same Laplace invariants. Therefore, it follows that

$$
\bar{A}=A, \bar{Q}_{s}=Q_{s}, s \neq i .
$$

We will show that $\bar{G}_{r}\left(x_{r}\right)=G_{r}\left(x_{r}\right)$. Substituting (3.39) in (3.40), we have

$$
\bar{V}=a V
$$

Since

$$
X=V\left[B_{j}-B_{k}\right], \bar{X}=\bar{V}\left[\bar{B}_{j}-\bar{B}_{k}\right] .
$$

Substituting (3.41), (3.42) and (3.43) in $\bar{X}=a X$ we have,

$$
\bar{B}_{j}-B_{j}-\left(\bar{B}_{k}-B_{k}\right)=0 .
$$

The same argument of item a) proves that $\bar{G} r\left(x_{r}\right)=G_{r}\left(x_{r}\right), \forall r$.

Example 1: Let $X: \mathbb{R}^{3} \rightarrow \mathbb{R}^{4}$ be an immersion

$$
X\left(x_{1}, x_{2}, x_{3}\right)=\left(\left(a+r \cos x_{1}\right) \cos x_{2},\left(a+r \cos x_{1}\right) \sin x_{2}, r \sin x_{1}, x_{3}\right),
$$

that describes a hypersurface of Dupin.

The principal curvatures are given by

$$
k_{1}=-\frac{1}{r}, \quad k_{2}=-\frac{\cos x_{1}}{a+r \cos x_{1}}, \quad k_{3}=0,
$$

From (2.4) we have that the laplace invariants are given by

$$
m_{i j}=0,1 \leq i \neq j \leq 3, m_{213}=-\frac{r \sin x_{1}}{a+r \cos x_{1}} \neq 0 .
$$

From Theorem 1, we get

$$
X=V\left[B_{2}-B_{3}\right] \text {, }
$$

where

$$
A=0, \quad V=-\frac{\left(a+r \cos x_{1}\right)^{2}}{a \sin x_{1}}, Q_{2}=1, \quad Q_{3}=a+r \cos x_{1} .
$$

The vectorial functions are given by

$$
\begin{aligned}
& G_{1}\left(x_{1}\right)=\left(0,0,-\frac{a}{\sin x_{1}\left(a+r \cos x_{1}\right)}, 0\right), \\
& G_{2}\left(x_{2}\right)=\left(\frac{a \cos x_{2}}{r}, \frac{a \sin x_{2}}{r}, 0,0\right), \\
& G_{3}\left(x_{3}\right)=\left(0,0,0,-\frac{a x_{3}}{r}\right) .
\end{aligned}
$$

Example 2: Let $Y\left(x_{1}, x_{2}\right)$ be a surface parametrized by lines of curvature on the sphere $\mathbb{S}^{3} \subset \mathbb{R}^{4}$ defined in $\Omega=I \times \mathbb{R}$, with two distinct principal curvatures $\widetilde{k}_{1}, \widetilde{k}_{2}$ that only depend on $x_{1}$ and satisfying $\widetilde{k}_{1,1} \neq 0, \widetilde{k}_{2,1} \neq 0, \forall x_{1} \in I, I$ is an open interval. We consider the hypersurface $X: \Omega \times J \rightarrow \mathbb{R}^{4}$ 
parametrized by lines of curvature given by $X\left(x_{1}, x_{2}, x_{3}\right)=Y\left(x_{1}, x_{2}\right)+x_{3} \widetilde{N}\left(x_{1}, x_{2}\right)$, where $\widetilde{N}$ is the normal vector of the surface $Y$ and $J$ is an open interval such that $\left(x_{3} \widetilde{k}_{i}-1\right) \neq 0, \forall x_{3} \in J, i=1,2$. We can show that the normal vector to $X$ is $Y$ and the distinct principal curvatures of $X$ are given by

$$
k_{1}=\frac{1}{x_{3} \widetilde{k}_{1}-1}, \quad k_{2}=\frac{1}{x_{3} \widetilde{k}_{2}-1}, \quad k_{3}=0
$$

By (2.3), we have

$$
\Gamma_{12}^{1}=\Gamma_{31}^{3}=\Gamma_{32}^{3}=0, \Gamma_{13}^{1}=\frac{\widetilde{k}_{1}}{x_{3} \widetilde{k}_{1}-1}, \Gamma_{21}^{2}=\frac{\left(x_{3} \widetilde{k}_{1}-1\right) \widetilde{k}_{2,1}}{\left(x_{3} \widetilde{k}_{2}-1\right)\left(\widetilde{k}_{1}-\widetilde{k}_{2}\right)}, \Gamma_{23}^{2}=\frac{\widetilde{k}_{2}}{x_{3} \widetilde{k}_{2}-1} .
$$

From (2.4) and (3.45) we have that the laplace invariants are given by

$$
\begin{gathered}
m_{12}=m_{21}=m_{23}=m_{31}=m_{32}=0, m_{13}=\frac{\widetilde{k}_{1,1}}{\left(x_{3} \widetilde{k}_{1}-1\right)^{2}}, \\
m_{213}=\frac{\left(x_{3} \widetilde{k}_{1}-1\right) \widetilde{k}_{2,1}}{\left(x_{3} \widetilde{k}_{2}-1\right)\left(\widetilde{k}_{1}-\widetilde{k}_{2}\right)}, m_{231}=\frac{\widetilde{k}_{1}-\widetilde{k}_{2}}{\left(x_{3} \widetilde{k}_{1}-1\right)\left(x_{3} \widetilde{k}_{2}-1\right)} .
\end{gathered}
$$

Also,

$$
C^{321}=\frac{x_{3} \widetilde{k}_{1}-1}{x_{3}\left(\widetilde{k}_{1}-\widetilde{k}_{2}\right)}
$$

From Theorem 1, we get

$$
X=V\left[B_{2}-B_{3}\right],
$$

where

$$
\begin{gathered}
A=-\frac{x_{3} \widetilde{k}_{2,1}}{x_{3} \widetilde{k}_{2}-1}+\frac{x_{3} \widetilde{k}_{1,1}}{x_{3} \widetilde{k}_{1}-1}+\frac{\widetilde{k}_{1,1}}{\widetilde{k}_{2}-\widetilde{k}_{1}}, \quad V=\frac{x_{3} \widetilde{k}_{1}-1}{\widetilde{k}_{1}-\widetilde{k}_{2}}, \\
Q_{2}=\frac{x_{3} \widetilde{k}_{1}-1}{x_{3} \widetilde{k}_{2}-1} e^{\int \frac{\widetilde{k}_{1,1}}{\widetilde{k}_{2}-\widetilde{k}_{1}} d x_{1}}, Q_{3}=\frac{x_{3} \widetilde{k}_{1}-1}{\widetilde{k}_{1}-\widetilde{k}_{2}} .
\end{gathered}
$$

The vectorial functions are given by

$$
\begin{aligned}
& G_{1}\left(x_{1}\right)=\left(-\widetilde{k}_{1,1}+2 \widetilde{k}_{2,1}+\frac{\left(\widetilde{k}_{1}-\widetilde{k}_{2}\right) \widetilde{k}_{2,11}}{\widetilde{k}_{2,1}}\right)\left(x_{1}\right) Y\left(x_{1}, x_{2}^{0}\right)+\left(\widetilde{k}_{2}-\widetilde{k}_{1}\right)\left(x_{1}\right) Y_{, 11}\left(x_{1}, x_{2}^{0}\right) \\
& G_{2}\left(x_{2}\right)=\left(\left(\widetilde{k}_{2}-\widetilde{k}_{1}\right) e^{\left.\int \frac{\widetilde{k}_{1,1}}{\widetilde{k}_{2}-\widetilde{k}_{1}} d x_{1}\right)\left(x_{1}^{0}\right) Y\left(x_{1}^{0}, x_{2}\right)}\right. \\
& G_{3}\left(x_{3}\right)=\left(a_{1} x_{3}+b_{1}, a_{2} x_{3}+b_{2}, a_{3} x_{3}+b_{3}\right),
\end{aligned}
$$

where

$$
\begin{aligned}
\left(a_{1}, a_{2}, a_{3}\right) & =\left(\frac{\widetilde{k}_{2}}{\widetilde{k}_{1}-\widetilde{k}_{2}} e^{-\int \frac{\widetilde{k}_{1,1}}{\widetilde{k}_{2}-\widetilde{k}_{1}} d x_{1}}\right)\left(x_{1}^{0}\right)\left[\left(\int \frac{G_{1}\left(x_{1}\right)\left(\widetilde{k}_{1}-\widetilde{k}_{2}\right) e^{-\int \frac{\widetilde{k}_{1,1}}{\bar{k}_{2}-\tilde{k}_{1}} d x_{1}}}{\widetilde{k}_{2,1}} d x_{1}\right)\left(x_{1}^{0}\right)\right. \\
& \left.+G_{2}\left(x_{2}^{0}\right)\right]-\widetilde{N}\left(x_{1}^{0}, x_{2}^{0}\right)-\left(\int \frac{G_{1}\left(x_{1}\right) \widetilde{k}_{2}}{\widetilde{k}_{2,1}} d x_{1}\right)\left(x_{1}^{0}\right) .
\end{aligned}
$$

We observed that this class of hypersurfaces has nonconstant Möbius curvature and they are not Dupin. 
4. Conclusions. From the results obtained in this work we can make the following conclusion: The fact of considering hypersurfaces parametrized by lines of curvature in $\mathbb{R}^{4}$ with Laplace invariants $m_{j i}=m_{k i}=0, m_{j i k} \neq 0$ for $i, j, k$ distinct fixed, allows us to find the same representation obtained in [15], therefore, this work generalizes the results obtained in [15], i.e., the hypersurfaces of Dupin studied in [15] are contained in this class of hypersurfaces.

\section{References}

[1] Cecil, T.E. and Chern, S.S. Dupin submanifolds in Lie sphere geometry, Differential geometry and topology 1 - 48, Lecture Notes in Math. vol. 1369, Springer, Berlin, Heidelberg, 1989.

[2] Cecil, T.E. and Ryan, P.J. Conformal geometry and the cyclides of Dupin, Can. J. Math. 1980; (32): 767 - 782

[3] Cecil, T.E. and Ryan, P.J. Tight and taut immersions of manifolds, Pitman, London, 1985.

[4] Chern, S.S. An introduction to Dupin submanifolds, Differential Geometry, 95 - 102, Pitman Monographs Surveys Pure Appl. Math. 52, 1991.

[5] Cecil, T.E. and Jensen, G. Dupin hypersurfaces with three principal curvatures, Invent. Math. 1998; (132): 121 - 178.

[6] Cecil, T.E. and Jensen, G. Dupin hypersurfaces with four principal curvatures, Geom. Dedicata, 2000; (79): 1 - 49.

[7] Kamran, N. and Tenenblat, K. Laplace transformation in higher dimensions, Duke Math. Journal 1996; (84): 237 - 266.

[8] Kamran, N. and Tenenblat, K. Periodic systems for the higher-dimensional Laplace transformation, Discrete and continuous dynamical systems, 1998; 359 - 378.

[9] Miyaoka, R. Compact Dupin hypersurfaces with three principal curvatures, Math. Z. 1984; (187): 433 - 452.

[10] Niebergall, R. Dupin hypersurfaces in $\mathbb{R}^{5}$, Geom. Dedicata 1991; (40): 1 - 22, and 1992; (41): 5 - 38.

[11] Pinkall, U. Dupinsche Hyperflachen, Dissertation, Univ. Freiburg, 1981.

[12] Pinkall, U. Dupinsche Hyperflachen in $E^{4}$, Manuscripta Math. 1985; (51): 89 - 119.

[13] Pinkall, U. Dupin hypersurfaces, Math. Ann. 1985; (270): 427 - 440.

[14] Riveros, C.M.C. A Characterization of Dupin hypersurfaces in $\mathbb{R}^{4}$, Bull. Belg. Math. Soc. Simon Stevin 2013 ; (20): 145 - 154.

[15] Riveros, C.M.C. Hipersuperfícies de Dupin em espaços Euclidianos, Dissertation, Univ. Brasília, 2001.

[16] Riveros, C.M.C. and Tenenblat, K. On four dimensional Dupin hypersurfaces in Euclidean space An. Acad. Bras. Cienc. 2003; 75(1): $1-7$.

[17] Riveros, C.M.C. and Tenenblat, K. Dupin hypersurfaces in $\mathbb{R}^{5}$. Canad. J. Math. Vol. 2005; $57(6)$ : 1291 - 1313.

[18] Stolz,S. Multiplicities of Dupin hypersurfaces, Invent.Math. 1999; (138): 253 - 279.

[19] Thorbergsson,G. Dupin hypersurfaces, Bull. London Math. Soc. 1983; (15): 493 - 498. 